\title{
Measure by measure, they touched heaven
}

\author{
Luisa Spairani ${ }^{1}$ \\ ${ }^{1}$ Gruppo Astrofili Eporediesi, C.so Vercelli 444, 10015 Ivrea, Italy
}

\begin{abstract}
The measure of distances is a recurring theme in astrophysics. The interpretation of the light coming from a luminous object in the sky can be very different depending on the distance of the object. Two stars or galaxies may each have a different real brightness, although they may look similar. The correct measures were determined by women computers a century ago. Special mention is due to Williamina Fleming, who supervised an observatory for 30 years and worked on the first system to classify stars by spectrum. Antonia Maury helped locate the first double star and developed a new star classification system. Henrietta Leavitt determined a law to calculate stellar distances. The most famous of the Harvard computers was Annie Jump Cannon. An expert in photography, she catalogued over 350,000 stars and expanded the classification system used today, but it was Henrietta Leavitt who left an indelible mark by discovering a law for the determination of stellar distances. In the same period, Italian women computers began to collaborate in observatories, but their tracks are obfuscated.
\end{abstract}

\section{Section: RESEARCH PAPER}

Keywords: women computers; IMEKO; Pickering; Cannon; Leavitt; Fleming; Maury

Citation: Luisa Spairani, Measure by Measure, they touched the heaven, Acta IMEKO, vol. 10, no. 1, article 35, March 2021, identifier: IMEKO-ACTA10 (2021)-01-35

Editor: Ioan Tudosa, University of Sannio, Italy

Received April 28, 2020; In final form November 19, 2020; Published March 2021

Copyright: This is an open-access article distributed under the terms of the Creative Commons Attribution 3.0 License, which permits unrestricted use, distribution, and reproduction in any medium, provided the original author and source are credited.

Corresponding author: Luisa Spairani, e-mail: luisa.spairani@netsurf.it

\section{INTRODUCTION}

Today, why do only women work in labs where electronic boards are prototyped? The reason is the precision that women generally apply - the same precision that was required from women computers for astronomical measures. In the early 1900s, women computers at Harvard Observatory went beyond performing calculations and discovered a new method for measuring the distances of the stars and to classify them. This method is still used today. The Harvard computers used Cepheids as distance markers. The important feature that allows a Cepheid variable to be used for distance measurements is that its period is directly related to its luminosity. This relation makes it possible to determine how much brighter than the Sun the star is.

The discovery of the connection between these variable stars and distance passed through several steps:

- Distances in astrophysics are notoriously difficult to calculate.

Visual astronomical measurements introduced subjective uncertainties. Photography overcame the problem of subjectivity by creating an enormous quantity of photos to analyse.
Women computers executed calculations, and measure by measure, they unveiled new laws that expanded the universe.

Women computers were not only active in the United States. There were female contributions in at least two Italian observatories during the period taken into consideration here (the end of the nineteenth century and the beginning of the twentieth).

\section{DISTANCES IN ASTROPHYSICS}

Distances in astrophysics are notoriously difficult to calculate. It is possible to use geometric methods to determine the distance of objects that are in the proximity of the solar system, i.e. within a distance of about 150 light-years from us. Beyond that, it is impossible to use any simple method to calculate distances. And this was the situation in astronomical research at the beginning of the last century. Many new objects had been discovered, but without knowing their distances, it was impossible to put them into any stellar systems model. At that time, before 1900, it was not known that we live in a galaxy called the Milky Way and that many other galaxies exist. 
In 1912, Henrietta Swan Leavitt, an American woman computer, elegantly removed this large handicap with a very important discovery: she found a way to determine the real brightness of a particular type of star. Yet Henrietta was not the only lady of the stars. The extent of this discovery can be evaluated by connecting three different elements: variable stars, astronomical photography and women computers.

\section{THE CEPHEIDS}

For the calculation of the distance of nearby stars, the geometric method consists in observing the star on two occasions, six months apart from each other (so that the Earth is in two opposite positions with respect to the Sun). The star, compared to the background composed of other more distant stars, will be in two different positions, and from the measure of the subtended angle, it is possible to derive the distance. But can we measure the remote stars that are in the background or those stars whose brightness varies?

The apparent brightness of a star is how bright it seems when viewed from the Earth, but a large, bright star can appear dim if it is a long way from the Earth, and a dim star can appear bright if it is close to the Earth. Therefore, the apparent magnitude has no bearing on the distance from the Earth. To give an accurate measurement of the brightness of a star, an absolute magnitude scale is needed.

Moreover, not all stars have a constant brightness over time: many of them show a variable brightness (the causes of variability are many). In 1784, during the time of Messier, a very young British amateur astronomer, John Goodricke, discovered that the Delta star of the constellation Cepheus was a variable star with a brightness that oscillated in a very regular manner. In fact, this star reaches a maximum brightness and then fades until it reaches a minimum; then the brightness begins to grow again until it reaches its maximum and so on, like a clock.

This particular type of variable star was called a Cepheid in honour of this constellation. Cepheids are very numerous, and above all, they are scattered everywhere; their period of change in brightness varies from a few hours to a few days and up to about two months. We now know that these variations are due to perturbations in the inner part of these stars and constitute a temporary phase of their life.

Until the mid-nineteenth century, every measure was meticulously performed based on the astronomer's observational abilities but with an unavoidable component of subjectivity, until it was possible to take photographs and thus have a database available to work with.

\section{ASTRONOMICAL PHOTOGRAPHY}

Between 1847 and 1852, the photographer John Adams Whipple and the astronomer William Cranch Bond, at the time director of Harvard College Observatory, used the observatory's large refracting telescope to produce images of the Moon of considerable detail and clarity. On July 16, 1850, Whipple and Bond made the first daguerreotype of a star: Vega. It was the beginning of astronomical photography. Although glass-plate photography was less sensitive than the eye, it had the advantage of having memory: what the eye does not see in a tenth of a second, it will never see; meanwhile, the photons that arrive at a given moment on the plate add their action to those that came before. That's why photography can employ long exposure times, which allow for the capture of more information.
In 1872, the first photograph of the spectrum of a star was obtained, and the study of stellar spectra opened a new frontier. Thus began the enormous work that Edward Pickering (18461919) undertook at Harvard College Observatory (HCO) in the United States. In this work, large prisms were used to disperse light and obtain the spectra of many stars at once. The Observatory organised a systematic observation of the brightest stars in both the Southern and the Northern Hemispheres. A large amount of data was collected even by today's standards, and the Harvard computers carried out much of the work of cataloguing and analysing that data. These computers were several dozen women who were hired to carry out the most tedious tasks of astronomical data analysis and paid about half as much as their male counterparts [1].

In 1928, astronomers worked on the spectra of 250,000 stars thanks to the measurements of women computers!

\section{WOMEN COMPUTERS}

As doctor and Harvard professor Edward Clarke wrote in his 1873 book Sex in Education, 'A woman's body could only handle a limited number of developmental tasks at one time - that girls who spent too much energy developing their minds during puberty would end up with undeveloped or diseased reproductive systems.'

For two centuries, the computers were mental workers who could efficiently grind logarithms. A large percentage of them were women. The male scientists of that time considered creative mathematics to be beyond feminine abilities but found women perfect for this type of numerical 'embroidery'. Calculation times were measured in 'girl hours': a complex calculation could even require 'kilo-girl-hours'.

Most human computers left no record of their personal life. One of the first was Nicole-Reine Lepaute, the wife of a watchmaker of the king of France. In 1750, together with two male colleagues, she foresaw the return of Halley's Comet in 1758 after an absence of 76 years. The estimation was incorrect by just one month.

Human computers learned to execute complex tasks. Someone cross-checked to screen errors, and a human computer called the comparator verified the work and searched for discrepancies.

As late as 1940, a giant mathematical table project was active, employing more than 300 human computers, half of whom used only paper and pencil. In 1952, IBM began selling its electronic computer model 701 for scientific activities. By 1960, calculating machines performed almost all numerical calculations.

Returning to the Harvard computers, the most remarkable woman computer was Miss Henrietta Swan Leavitt (1868-1921). The daughter of a Congregationalist minister, she graduated from Radcliffe College and was hired as a computer at HCO in 1880. Her job was to compare photographic plates of the Magellanic Clouds to detect small differences in the brightness of the stars. Now we know these entities are neighbouring galaxies, the companions of our Milky Way. At that time, no one was quite sure what they were. Bent over the plates in an observatory laboratory, Miss Leavitt found the model that led to the answer. She discovered a way to make measurements beyond our galaxy and begin to map the universe [2].

Leavitt determined that some stars have a consistent brightness no matter where they are located, making it easy to figure out their distance from Earth.

The visual brightness of a star depends on its size or its distance from the Earth: stars with similar apparent magnitudes 
TABLE 1 .

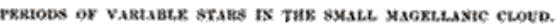

\begin{tabular}{|c|c|c|c|c|c|c|c|c|c|c|c|c|c|}
\hline n. & Mas. & xis. & nemat & Wrate & $\sin x$ & ren. & n. & $\operatorname{Mex}$ & xtion & nater. & Nerest. & $\ln , x_{0}$ & then an \\
\hline 1505 & 14.8 & 16.1 & 0.02 & 1.2535 .5 & -0.6 & -0.5 & two & U.1 & 148 & ${ }_{40}^{2}$ & 6.600 & +0. & -0.3 \\
\hline 1436 & 14.8 & 16.4 & 0.02 & 1.6637 & -0.3 & +0.1 & t3565 & 14.0 & 14.8 & 4.8 & 7,483 & $\begin{array}{l}1 \\
+0.2\end{array}$ & -0.2 \\
\hline 1446 & 16.8 & 16.4 & 1.38 & 1.7620 & -0.3 & +0.1 & 1374 & 13.9 & 15.2 & 6.0 & 8.397 & +0.2 & -0.3 \\
\hline 1506 & 15,1 & 163 & 1.08 & 187502 & $+a .1$ & +0.1 & 818 & 136 & 14.7 & 40 & 10.336 & 0.0 & 0.0 \\
\hline 1413 & 14.7 & 15.6 & 0.35 & 2.17352 & -0.2 & -0.3 & 1610 & 13.4 & 14.6 & 11.0 & 11.645 & 0.0 & 0.0 \\
\hline 1460 & 14.4 & 15.7 & 0.00 & 2.913 & -0.3 & -0.1 & 1385 & 13,8 & 14.8 & 9.6 & 12,417 & +0.4 & +0.2 \\
\hline 1429 & 14.7 & 15.9 & 0.6 & 3.501 & +0.2 & +0.2 & 135t & 13.4 & 144 & 40 & 12.08 & $+0,1$ & -0.1 \\
\hline$\$ 42$ & 14.6 & 16.1 & 2.61 & 4.2897 & +0.3 & +0.6 & 827 & 13.4 & 14.3 & 11.6 & 13.47 & +0.1 & -0.2 \\
\hline 1625 & 14.3 & 15.3. & 28 & 4.512 & 0.0 & -0.1 & sez & 13.0 & 14.6 & 130 & 16.73 & -0.1 & +0.3 \\
\hline 1742 & 14.3 & 15.5 & 0.95 & 4.9866 & +0.1 & $+c, 2$ & 823 & 122 & 14.1 & 2.9 & 31.94 & -0.3 & $+a x$ \\
\hline 1646 & 14.4 & 15.4 & 4.30 & 3.311 & +0.3 & +0.1 & 824 & 11.4 & 12.8 & 4. & 65.8 & -0.4 & -0.2 \\
\hline 1649 & 14.3 & 15.2 & 5.05 & 5.323 & +0.2 & -0.1 & $\$ 21$ & 11.2 & 12.1 & 97. & 127.0 & -0.1 & $-0,4$ \\
\hline 1492 & 13.8 & 14.8 & 0.6 & 62.2026 & -0.2 & -04 & & & & & & & \\
\hline
\end{tabular}

Figure 1. Periods of variable stars published by Harvard College Observatory - Circular 173 - Edward C. Pickering, March 3, 1912.

could be at different distances. But Leavitt had also noticed that variable stars emit pulses of light and that the true brightness of a star can be measured by the speed of its pulses. Brighter stars flash more slowly. By comparing the length of time between pulses with the apparent brightness of the star, one can estimate how far away it is.

The astronomers were impressed. 'What a variable star "friend" Miss Leavitt is. One can't keep up with the roll of the new discoveries', wrote a Princeton astronomer in a letter to his boss.

Although she was praised for her excellent work, Miss Leavitt earned neither a promotion nor any privilege to pursue her research. She quietly continued her human computer activities, remaining single and living a life appropriate for a distinguished Bostonian young lady.

Even her emotions about her important discovery remain a mystery; she left no diaries or letters.

Yet her words witness her commitment.

The discovery of variable stars, at this Observatory and elsewhere, has progressed so rapidly during the last five years, that the difficulty of keeping pace in observing and discussing them has become very great. In the study of distribution now in progress here, the actual time devoted to the search for new variables is small, but thorough observation requires much time, while the discussion of results may be prolonged almost indefinitely

A remarkable relation between the brightness of these [Cepheid] variables and the length of their periods will be noticed.

Since the [Cepheid] variables are probably at nearly the same distance from the Earth, their periods are apparently associated with their actual emission of light, as determined by their mass, density, and surface brightness.

It is hoped that systematic study of the light changes of all the variables, nearly two thousand in number, in the two Magellanic Clouds may soon be undertaken at this Observatory.'

Other women computers left written testimonies that sometimes expressed their frustration because they were not allowed to explore the deeper implications of what they had found.

Of course, not all human computers were women. Some were men, mostly young and looking for a stable job: they were mathematicians, not scientists. But it was the women computers who left a legacy. [3].

Harvard computers became known as 'the Pickering harem'

This expression illustrates the valuation of their work. More than 80 women worked for Pickering at the Harvard Observatory, putting in six-day weeks poring over photographs and earning 25 to 50 cents an hour (half what a man would have

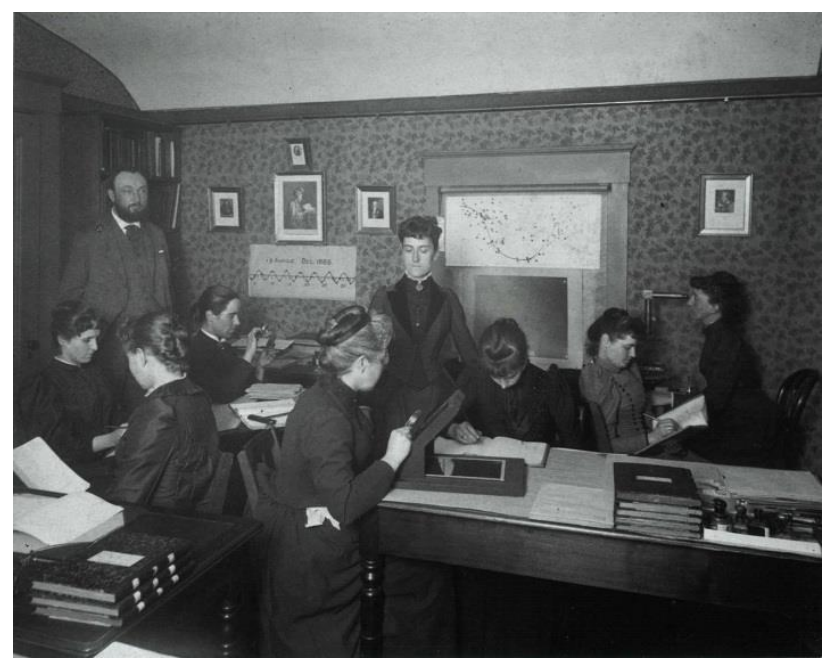

Figure 2. Harvard computers, courtesy of Harvard-Smithsonian Center for Astrophysics' Wolbach Library.

been paid). The daily work was largely clerical. Some women would reduce the photographs, taking into account things like atmospheric refraction, to render the image as clear and unadulterated as possible. Others would classify the stars by comparing the photographs to known catalogues. Others catalogued the photographs themselves, making careful notes of each image's date of exposure and the region of the sky. The notes were then meticulously copied into tables, which included the star's location in the sky and its magnitude.

In 1901, the director of Yale Observatory, William Elkin, said: 'I am thoroughly in favour of employing women as measurers and computers. Not only are women available at smaller salaries than are men, but for routine work they have important advantages. Men are more likely to grow impatient after the novelty of the work has worn off and would be harder to retain for that reason.'

Many of the women were bright and inquisitive and quickly expanded their roles. Williamina Paton Stevens Fleming (18571911) [4], who headed the group, discovered the Horsehead Nebula [5]. It is worth mentioning that she first worked as a maid in the home of Professor Pickering after being abandoned, pregnant, by her husband.

Williamina wrote:

'A large nebulosity extending nearly south from Zeta Orionis for about 60 minutes. More intense and well marked on the following side, with a semicircular indentation 5 minutes in diameter 30 minutes from Zeta.' (Annals of the Harvard College Observatory, 18 (1890) p. 116)

'In the Astrophotographic building of the Observatory, 12 women, including myself, are engaged in the care of the photographs.... From day to day my duties at the Observatory are so nearly alike that there will be little to describe outside ordinary routine work of measurement, examination of photographs, and of work involved in the reduction of these observations.'

Before lunch I found time to examine a few southern spectrum plates and marked a fourth type star and a gaseous nebula, both probably known. Later in the afternoon I noted a few more interesting objects, among these two fourth type stars, one gaseous nebula, and several bright line stars. Some of these may be new.' 
The entries also provide insight into her dedication and the sheer workload. Looking after the numerous pieces of routine work which have to be kept progressing, searching for confirmation of objects discovered elsewhere, attending to scientific correspondence, getting material in form for publication, etc, has consumed so much of my time during the past four years that little is left for the particular investigations in which I am especially interested,' she wrote.

Williamina expresses the same frustrations as current astronomers - that project management consumes more time than research. The Director, however, says that my time employed in the above work is of more value to the Observatory so I have delegated my measures of variables etc to Miss Leland and Miss Breslin. I hope, however, to be able soon to finish the measures of the out of focus plates and to get well settled down to my general classification of faint spectra for the new Draper catalogue."

A few, like Miss Leavitt, looked up at the stars and turned the job of human computer into something much greater.

Many other women at HCO made history through astronomical research. In particular, Annie Jump Cannon (18631941), who was originally hired to do mathematical calculations by hand and examine photographs, was able to make a great contribution in the field of stellar classification. Other women that deserve mention are Antonia Maury (1896-1952) and Cecilia Payne-Gaposchkin (1900-1979). They discovered hundreds of variable stars and many novae; these last were revealed through analysis of the enlarged emission lines of their spectrum [6], [7], [8], [9].

They introduced a new classification of stellar spectra that included most of the stars and was related to the stars' physical properties.

In 1912, an extraordinary year, Annie Cannon described the sequence of spectral types in the article 'Classification of 1477 stars by means of their photographic spectra' [10]; for mnemonic use, she invented the phrase 'Oh Be A Fine Girl, Kiss Me'. In substance, this mnemonic is still in use today, O B A F G K M.

Angelo Secchi, a Jesuit priest and a scientist (1818-1878), first distinguished four main spectral types of stars. At HCO in the 1880s, during the compilation of the Henry Draper Catalogue of stars [11], more types were distinguished and were designated by letters in an alphabetic sequence from $\mathrm{A}$ to $\mathrm{P}$ according to the strength of their hydrogen absorption spectral lines. The modern Cannon system eliminated many classes and reordered the remaining ones, creating a sequence dependent on the effective surface temperature of the stars. The classes are still in use:

O: $30,000-60,000 \mathrm{~K}$ blue stars

B: $10,000-30,000 \mathrm{~K}$ blue-white stars

$$
\begin{aligned}
& \text { A: 7,500-10,000 K white-stars } \\
& \text { F: 6,000-7,500 K yellow-white stars } \\
& \text { G: 5,000-6,000 K yellow stars (like our Sun) } \\
& \text { K: 3,500 - 5,000 K light orange stars }
\end{aligned}
$$

\section{$\mathrm{M}:<3,500 \mathrm{~K}$ orange stars}

Since its creation, 3 new classes have been added: N, R and S, which are darker orange and colder.

\subsection{The law of Leavitt}

The absolute magnitude ( $M$, also called absolute luminosity) is the apparent magnitude $(m)$ that an object would have if it were

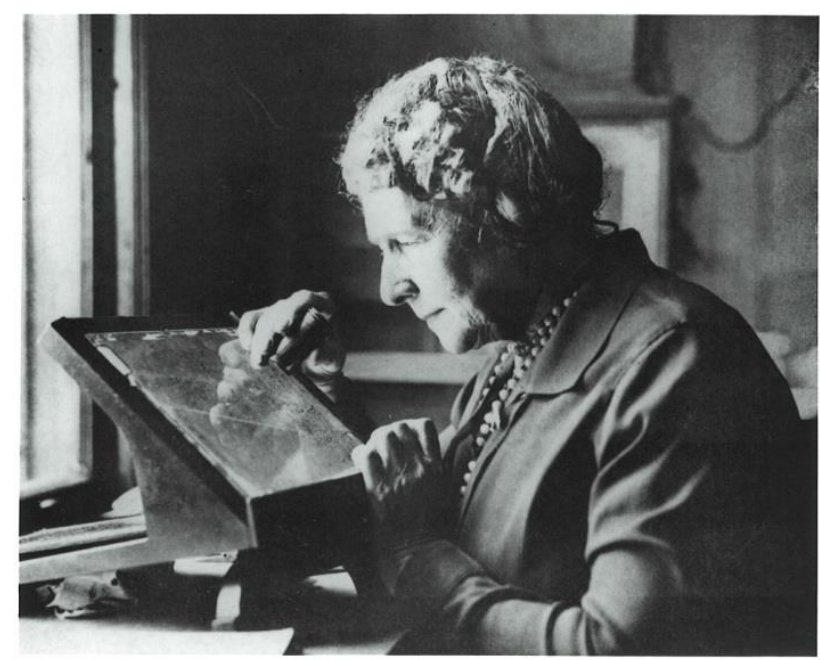

Figure 3. Annie Cannon with plate, courtesy of Harvard-Smithsonian Center for Astrophysics' Wolbach Library.

at a distance of 10 parsecs (32.616 light-years) or $3 \times 10^{14}$ kilometres from the observer. Leavitt's law expresses the dependence of the absolute magnitude on the apparent one and presents the logarithm of the distance:

$$
M=m+5-5 \log (d),
$$

where $m$ is the apparent magnitude and $d$ is the real distance of the star expressed in parsecs.

Leavitt's discovery made it possible to calculate distances between 100 and 10 million light-years, thus paving the way for the fundamental work of astronomers Hubble and Shapley, which revolutionised our knowledge of the galaxy and the universe, while the period-luminosity relationship identified in the Cepheids represents the foundation of our current extragalactic distance scale.

Throughout her career, Leavitt discovered over 2,400 variable stars and provided the basis for scientists to further research and learn more about the cosmos. Leavitt continued to work at Harvard College Observatory until 1921, when she died of cancer at the age of 53 .

Perhaps in part because of this premature death, her name did not receive the recognition she deserved in the scientific community of the time. She was proposed as a Nobel Prize winner four years after her death by a committee member who did not know of her untimely end, so the award went to the

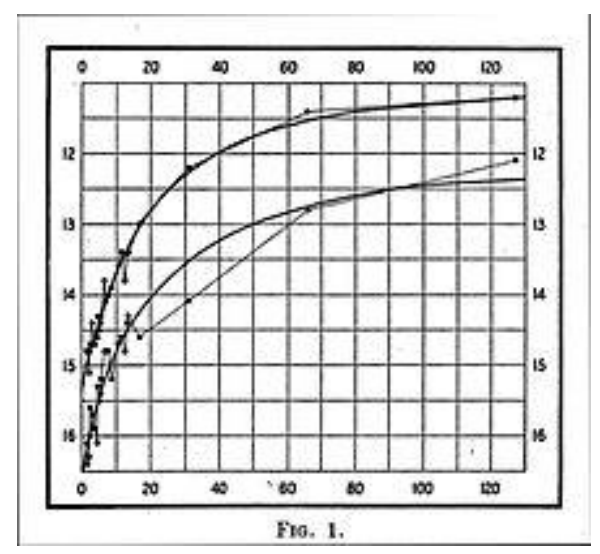

Figure 4. 'Periods of 25 Variable Stars in the Small Magellanic Cloud' Harvard College Observatory Circular, vol. 173. From Wikimedia Commons. 
astronomer Harlow Shapley, head of the Harvard College Observatory (1921-1952).

The importance of her law was immediately appreciated, however. A year after the discovery, the Danish astronomer Ejnar Hertzsprung (1873-1967) determined the distances of some Cepheids in the proximity of our Milky Way using geometric methods. (Today we know that this measure also depends on some factors that were unknown at that time. For example, we realised later that the measure depends in some way on the quantity of heavy elements in the stars.)

Within the next 5 years, Shapley applied Leavitt's law to globular clusters and was able to produce the first map of the Milky Way. He made a model of a vast system of stars - about a hundred billion, structured in a disc - the centre of which is far from our solar system. Shapley had created a whole new chapter in astronomy, and this was only possible because of Leavitt's crucial discovery.

Since then, astronomers have devised a series of further relationships between the properties of different astronomical objects (supernovae and HII regions, i.e. regions of interstellar atomic hydrogen that is ionised, just to name a few) that can be used to determine even greater distances of objects in places where Cepheids are difficult to locate. The period-luminosity relationship of Cepheids, however, remains the least uncertain method of determining distances.

In 1924, astronomer Edwin P. Hubble (1889-1953), using the new $254 \mathrm{~cm}$ diameter Mount Wilson telescope (at that time the largest in the world), was able to resolve the outer parts of the Andromeda Nebula and discover dozens of Cepheids. Based on the (very reasonable) hypothesis that the Cepheids of the Andromeda Nebula behave like those of our galaxy, it was finally possible to calculate the distance of the Nebula. The result made the astronomers pale: the Nebula is between 700,000 and $1,000,000$ light-years away, which, for those times, was an immense distance.

Furthermore, the application of Leavitt's law allowed for the classification of celestial phenomena previously seen as spots in the night sky and provided substantial evidence that the universe is expanding.

\subsection{Leavitt's legacy}

One of the key projects of the Hubble Space Telescope, launched more than a decade ago, was the identification of the Cepheid variable stars in the Virgo Cluster (the nearest galaxy cluster in the Milky Way, at a distance of about 50 million lightyears) to determine their distance more precisely. Cepheids are the most useful stars in the sky; they are the 'standard candles'.

Even today, a century later, thanks to the fundamental contribution of Henrietta Leavitt, Cepheid variable stars remain a reference in astronomical research for the estimation of cosmological distances.

\section{Italian women computers}

During the same period as the Harvard computers, in the young Italian nation, women made important contributions at the Observatory of Turin and the Vatican Observatory.

\subsection{At the Turin Observatory}

The Observatory of Turin, founded in 1759, moved from the roofs of Palazzo Madama in the town up to the hill in 1913. As early as 1904 , women astronomers were contributing.
'... The Faculty of Mathematics of Turin is attended by quite a few young ladies, some of whom have a real aptitude for those severe studies. If then the post of assistant to the chairs of the first two years were given by competition, this would easily be won by one of these doctors. Now, would it be prudent to put as a teaching assistant, for example in descriptive geometry and corresponding drawing, a young girl among 180 students?'

This question came from Father Giovanni Boccardi, the director of the Astronomical Observatory of Turin at the beginning of the twentieth century.

He hired women computers. Their work consisted of the reduction of observational data. Moreover, the staff also began to run low, even before the First World War, beginning in 1911 with the war in Tripolitania. The director of the Observatory noted the exodus of assistant astronomers called to military service, so, in the shortage of trained professionals and funds, Boccardi availed himself of the help of undergraduate women.

Luisa Viriglio was on staff from 1904 to 1906. The volunteer assistants received a much lower salary than those at higher levels.

Despite the short amount of time she spent working at Palazzo Madama, Viriglio holds another record in addition to being the first salaried astronomer. She was also the first Italian female astronomer to have a publication in her name. Other mathematicians employed in the period were Ernesta Fasciotti and Giovanna Greggi.

The 'Essays of Popular Astronomy', scientific bulletins published by the astronomical society Urania, first appeared in January 1911. The years 1914 and 1915 even saw the simultaneous presence of three female graduate assistants in mathematics. Teresa Castelli and Tiziana Comi flanked the work of Dr Greggi.

Tiziana Comi and Jeannette Mongini carried out dissemination activities.

In 1919-1920, Corinna (or Carolina) Gualfredo was listed among the staff as a custodian but also as an astronomer.

All that remains of them are their names and surnames, graduation marks and salary records as well as the numbers they calculated patiently by hand. But we do not even have a portrait in an ancient photograph from the beginning of the century [12].

\subsection{At the Specola Vaticana}

In the early decades of the twentieth century, four nuns from the Suore di Maria Bambina Institute contributed to a catalogue of 400 thousand stars, which is still in the preserve of international stellar 'cartography'.

Their names are Emilia Ponzoni, Regina Colombo, Concetta Finardi and Luigia Panzeri. The story begins in the late nineteenth century, in Paris. The French capital hosted astronomers from all over the world with a prestigious goal: to create the largest chart of the sky in order to worthily represent the celestial vault. The Vatican also took part, led by Pope Leo XIII, who was passionate about stars.

Representing the Pope was the priest Francesco Denza, who made an agreement with the Jesuit John Hagen, from the United States. The latter confided to the Italian the need for helpers who knew how to 'read' the celestial coordinates and therefore the positions of the stars. Don Francesco passed the work into the hands of four nuns employed at the Vatican Observatory who have remained anonymous until recently. The nuns learned the art of observing the stars and within a few years became experts in the starry world. They learned to manoeuvre the telescope and to extricate themselves from complicated mathematical calculations; they were women computers. 
The famous Carte du Ciel was concluded in 1966 and included the identification of five million stars, many of which were reported for the first time by four silent and devoted Roman nuns [13].

\section{AT THE END}

Fictional literature introduced computational devices when women computers were leading the field. 'The Machine Stops' (1909) by E. M. Forster is a short story about the role of technology in our lives, and twentieth century fiction was full of this topic in the works of Heinlein, Asimov, van Vogt and many others. The success and the limits of manual computing were a source of inspiration for writers of the positivism period. Camille Flammarion, who began his career as a human computer, mentioned computer devices in his book The Planet Mars. Did the human computers' scientific efforts influence the vision of the future?

We cannot learn how to solve specific scientific problems from history, but we can come to better understand our contemporary science and frame it within its social context.

'By the serious attempt to put ourselves back into the intellectual situation of the ancient thinkers, far less experienced as regards the actual behaviour of the nature, but also very often much less biased, we may regain from them the freedom of thought' [14].

\section{ACKNOWLEDGEMENT}

I would like to thank Dr Antonella Bianchi (Biblioteque Bocconi University) for helping me with the presentation of this paper at the 2019 IMEKO TC4 International Conference on Metrology for Archaeology and Cultural Heritage in Florence, Italy, December 4-6, 2019.

I would also like to thank Drs F. Bonoli, I. Chinnici, D. Randazzo and V. Zanini of the National Institute for Astrophysics [15] for their support with research about female Italian astronomers.

\section{REFERENCES}

[1] L. Spairani, Henrietta Leavitt e la misura dell'Universo, Le Stelle X (2011), pp. 68-73.

[2] D. Sobel, The Glass Universe, Viking, New York, 2016, ISBN 9780698148697, p. 9.

[3] N. Geiling, The women who mapped the universe and still couldn't get any respect, Smithsonian Magazine, Sept. 18 (2013). Online [Accessed 19 March 2021]

https://www.smithsonianmag.com/history/the-women-who- mapped-the-universe-and-still-couldnt-get-any-respect9287444/\#0bLccaAHH

[4] Harvard University Library - Open Collection Programs, Women Working, 1800-1930. Online [Accessed 19 March 2021] http://ocp.hul.harvard.edu/ww/fleming.html

[5] A. McGrath The first computer: Williamina Fleming and the Horsehead Nebula, Galactic Gazette July 3 (2017). Online [Accessed 19 March 2021]

https://web.archive.org/web/20190929142207/http:/altbibl.io/ gazette/the-first-computer-williamina-fleming-and-thehorsehead-nebula/

[6] Wolbach Library, Historical Figure Index, Antonia Maury, Online [Accessed 19 March 2021] https://library.cfa.harvard.edu/phaedra/maury https://library.cfa.harvard.edu/redshift-spectroscopy.

[7] C. P. Gaposchkin, On the physical condition of the supernovae, Proceedings of the National Academy of Sciences 22(6) (1936), pp. 332-336.

[8] F. L. Whipple, C. P. Gaposchkin, On the bright line spectrum of Nova Herculis, Proceedings of the National Academy of Sciences 22(4) (1936), pp. 195-200.

[9] J. J. O'Connor, E. F. Robertson, Cecilia Helena PayneGaposchkin, University of St. Andrews. Online [Accessed 19 March 2021] http://www-groups.dcs.stand.ac.uk/history/Biographies/Payne-Gaposchkin.html

[10] A. J. Cannon, Classification of 1477 stars by means of their photographic spectra, Harvard Obs. Annals 56 (1912), pp. 65-114, in: Cannon, Annie Jump, 1863-1941. Papers of Annie Jump Cannon: an inventory, Harvard University Archives. Online [Accessed 19 March 2021] https://web.archive.org/web/20100714141345/http:/oasis.lib.h arvard.edu/oasis/deliver/ hua12001

[11] The Draper Catalogue of Stellar Spectra Photographed with the 8inch Bache Telescope as a Part of the Henry Draper Memorial, Cambridge University Press, Cambridge, 1890. Online [Accessed 19 March 2021] https://archive.org/details/drapercatalogueo00harvrich/page/n $\underline{8}$

[12] G. Bernardi, A. Vecchiato, The advent of female astronomers at Turin Observatory, The Journal of Astronomical History and Heritage 21(1) (2018), pp. 13-28. Online [Accessed 19 March 2021] https://www.academia.edu/36686097/THE ADVENT OF F EMALE ASTRONOMERS AT TURIN OBSERVATORY

[13] S. Sesti, L. Moro, Scienziate nel Tempo. Più di 100 Biografie, Ledizioni LediPublishing, Milano, 2018, ISBN 9788867057733.

[14] E. Schrödinger, Nature and the Greeks and Science and Humanism, Cambridge University Press, Cambridge, 1996 (1954) ISBN-13 978-0521575508.

[15] INAF, Polvere di stelle. Online [Accessed 19 March 2021] http://www.beniculturali.inaf.it/ 